\title{
ORBITS OF THE MAGELLANIC CLOUDS
}

\author{
SLOBODAN NINKOVIC \\ Astronomical Observatory of Belgrade \\ Belgrade, Volgina 7 \\ Yugoslavia \\ MIROSLAV FILIPOVIC \\ Science Centre of Petnica \\ c/-Astronomical Observatory of Belgrade \\ Belgrade, Volgina 7 \\ Yugoslavia
}

\begin{abstract}
We use a simple, spherically symmetric model for the Galaxy based on the rotation curve for the inner parts. We extrapolate beyond the rotation curve boundary, assuming a dark corona whose total mass is a model parameter. For each mass value assume we calculate the allowed angular-momentum interval for which a cloud is neither tidally disrupted, nor escapes. On the basis of this investigation we reach a conclusion about the expected mass of the Galaxy.
\end{abstract}

\section{Introduction}

Our own small galaxies-satellites play an important role in studies of the outer parts of the Milky Way, and because of the superior quality of the observational data the Magellanic Clouds (MCs) have a special place among them. In former studies the relatively small moduli of their galactocentric radial velocities and their consequences have been clearly pointed out (e.g. LyndenBell et al. 1983, Little \& Tremaine 1987). Therefore, our paper reexamines the motion of the MCs with respect to the Galaxy and compares the implications with the constraints on the total mass of the Milky Way following on from other kinds of evidence.

\section{Method}

Observational data on the MCs such as heliocentric distance, line-of-sight velocity, total mass and diameter are assumed according to Kulikovskij (1985 - table, p. 259). For the galactocentric distance of the Sun and the velocity of the Local Standard of Rest around the galactic centre we assume the values of $8.5 \mathrm{kpc}$ and $220 \mathrm{~km} \mathrm{~s}^{-1}$ respectively, following the IAU. In this way we obtain $51.5 \mathrm{kpc}$ and $63 \mathrm{kpc}$ for the galactocentric distances of the Large and Small Clouds respectively, i.e. $+89 \mathrm{~km} \mathrm{~s}^{-1}$ (LMC) and $+36 \mathrm{~km} \mathrm{~s}^{-1}$ (SMC) for the radial components of their galactocentric velocities.

We do not know the transverse components and at present we have no way of finding them; we can only estimate. For simplicity we assume spherical symmetry for our Galaxy and since, then, the angular momentum is constant, estimating the transverse components is reduced to estimating the angular momentum. For each of the Clouds one can specify the upper and lower 
limits of the angular momentum. These limits are valid for a particular value of the total mass of the Milky Way. The upper limit is defined through the requirement that the total mechanical energies of the Clouds are negative, since they are expected to be satellites of the Milky Way. This theoretical requirement may be followed by a more concrete one - that the apogalactic distances of the Magellanic Clouds have a reasonable upper limit, say $200 \mathrm{kpc}$. The choice of this value cannot be seriously verified at present but it seems reasonable, since the galactocentric distance of $200 \mathrm{kpc}$ appears to be a limiting one for almost all the satellites of our Galaxy (from data presented by Lynden-Bell et al. 1983). We specified the lower limit requiring that the tidal radius of each Cloud be not smaller than its optical radius (radius established from observations). The tidal radius is calculated according to King (1962 - equ. (7)).

The dependence of the galactic mass on the galactocentric radius we obtain by "spherization" of a galactic model based on the rotation curve. We assumed Haud's (1979) rotation curve interior to the Sun and that by Petrovskaya and Teerikorpi (1986) beyond the Sun. The spherization means that the values of the rotation velocity serve us as the values of the circular velocity in a spherically symmetric model. In this way we obtain the mass and the potential dependences on the galactocentric radius. The full value of the potential at any galactocentric distance we determine from assumed total mass of the Galaxy. The difference between the upper and lower limits of the angular momentum is the allowed angular-momentum interval. the minimal value of the galactic total mass is that for which the angular-momentum limits coincide. Thus we obtain that the "minimal mass of the Galaxy" in the case of the SMC is equal to only $6.6 \times 10^{10} \mathrm{M}_{\odot}$ and $10^{11} \mathrm{M}_{\odot}$ for the LMC The radii within which these masses are contained we obtain from the assumed circular velocities; in both cases they are smaller than the perigalactic distances of the Clouds. Mass values as small as the two mentioned above cannot be expected on the basis of the rotation curve, since it is likely that a flat rotation curve for the Galaxy is present to at lease double the distance between the Sun and Milky Way centre (Fich et al. 1989), or $17 \mathrm{kpc}$ in our distance scale. The extrapolation of the rotation curve assumed by us to $17 \mathrm{kpc}$ yields a total mass of the Galaxy of $1.88 \times 10^{11} \mathrm{M}_{\odot}$. With this total mass the allowed angular-momentum intervals become fairly broad for both Clouds and their orbits are sufficiently eccentric. The broadening becomes enhanced when a higher total galactic mass is assumed.

\section{The case of the fixed tidal radius}

The tidal radii of the Magellanic Clouds are not well known. However, the consequences are interesting if we fix them to their lower limits, i.e. to the values of the optical radii. The values of the optical radii we assumed are $3.5 \mathrm{kpc}$ for the LMC and $1.5 \mathrm{kpc}$ for the SMC (Kulikovskij 1985). Together with the mass values (assumed from the same source) they determine the perigalactic distance for a specified angular-momentum value. This angular momentum cannot be arbitrarily large. In our assumed framework of the galactic model there is a maximal galactic mass contained within each of the Clouds. This mass we determine easily by allowing the circular velocity to remain constant also beyond $17 \mathrm{kpc}$, to as far as the present distance of one of the Clouds. The value of the angular momentum, yielding the tidal radius assumed above in these conditions, is the maximal value still allowed in our model. Any other angular-momentum value smaller than this, will result in a smaller galactic mass within a Cloud (tidal radius fixed!) since then the difference of the galactic potentials at the perigalacticon and at the present Cloud distance, obtained by using the energy integral, will be smaller than that corresponding to the maximal mass. We find that the maximal masses still allowed in our model are equal to $5.7 \times 10^{11} \mathrm{M}_{\odot}(\mathrm{LMC})$ and $6.9 \times 10^{11} \mathrm{M}_{\Theta}$ (SMC). The maximal values of the angular momenta are: $4680 \mathrm{kpc} \mathrm{km} \mathrm{s}^{-1}$ (LMC) and $2080 \mathrm{kpc}$ 
$\mathrm{km} \mathrm{s}^{-1}$ (SMC). The corresponding values of the transverse velocities are $90 \mathrm{~km} \mathrm{~s}^{-1}$ (LMC) and 34 $\mathrm{km} \mathrm{s}^{-1}$ (SMC).

Though these values obtained here are the maximal ones within the limits of our model, one should not accept them as such since the tidal radii are very uncertain. We can only add that the neighbouring spiral galaxy-Andromeda Nebula (AN)- has also two close companions, M 32 and NGC 205, and from their line-of-sight velocities (Kulikovskij 1985) we find their residual velocities along the heliocentric direction to AN of $+87 \mathrm{~km} \mathrm{~s}^{-1}$ and $+58 \mathrm{~km} \mathrm{~s}^{-1}$, respectively. Bearing in mind the position of the triangle AN-Sun-Companion it is not difficult to see that these values are parts of the transverse components, with respect to the AN centre. They seem comparable to the values for the MCs mentioned above.

Therefore, it seems that the MCs move around the galactic centre along sufficiently eccentric orbits with low angular momenta. Such a conclusion is more similar to the findings of Little and Tremaine (1987), than to those of Lynden-Bell et al.(1983), who argue in favour of less eccentric orbits because of the possibility of tidal disruption. For example, the values of the transverse velocities mentioned above combined with the radial ones yield kinetic energies per unit mass of $8101 \mathrm{~km}^{2} \mathrm{~s}^{-2}$ (LMC) and $1226 \mathrm{~km}^{2} \mathrm{~s}^{-2}$ (SMC). Their doubled values correspond to the so-called "virial masses" of the Galaxy of $1.8 \times 10^{11}$ and $3.4 \times 10^{10} \mathrm{M}_{\odot}$, respectively.

\section{References}

Fich, M., Blitz, L \& Stark, A.A. (1989), Astrophys. J. 342, 272.

Haud, U.A. (1979), Pis'ma v. Astron. Zh. 5, 124.

King, I. (1962), Astron. J. 67, 471.

Kulikovskij, P.G. (1985), zvezdnaya astronomiya, Nauka, Glav. red. fiz.-mat.lit., Moskava.

Little, B., Tremaine, S. (1987), Astrophys. J., 320, 493.

Lynden-Bell, D., Cannon, R.D., Godwin, P.J. (1983), Mon. Not. R. Astr. Soc. 204, 87P.

Petrovskaya, I.V., Teerikorpi, P. (1986), Astron. Astrophys., 163, 39. 